\title{
Effect of Vitamin D Supplementation on Insulin, Fasting Blood Glucose, and Waist-Hip Ratio in Young Females with Pre-existing Vitamin D Deficiency
}

\author{
Mona Hmoud AlSheikh ${ }^{1, *}$, Shayma Ibrahim Almubayadh ${ }^{2}$ \\ ${ }^{1}$ Department of Physiology, College of Medicine, Imam Abdulrahman Bin Faisal University, Dammam, Saudi Arabia \\ ${ }^{2}$ College of Medicine, Imam Abdulrahman Bin Faisal University, Dammam, Saudi Arabia \\ *Corresponding author. E-mail: monaalsheikh@gmail.com
}

Received date: Jul 1, 2018; Revised date: Sep 16, 2018; Accepted date: Sep 19, 2018

\section{Abstract}

$\mathrm{B}$

ACKGROUND: Vitamin D is an essential nutrient requirement for adults and children for normal metabolism and maintenance of healthy bones. Its deficiency may disrupt homeostasis and contribute to systemic diseases, especially in obese patients. This study aimed to examine the effect of vitamin D on insulin, fasting blood glucose (FBG) and waist-hip ratio (WHR) in young and obese Saudi females with pre-existing hypovitaminosis D.

METHODS: The results of this study were based on prospective parallel randomised controlled clinical trial conducted at King Fahd University Hospital in which 30 obese Saudi young females aging 18-23 years with preexisting vitamin $\mathrm{D}$ deficiency contributed. Participants were randomly assigned to either vitamin D supplementation group (experimental) or placebo group (control). Subjects of vitamin D group received 50,000 IU/week of cholecalciferol drops and placebo group received $10 \mathrm{~mL}$ of normal saline drops for 12 weeks.

\section{Introduction}

Vitamin D refers to a group of fat-soluble secosteroids, of which, the most important compounds are vitamin D3 (cholecalciferol) and vitamin D2 (ergocalciferol). Vitamin $\mathrm{D}$ is primarily responsible for the intestinal absorption, homeostasis and metabolism of calcium in the body, which is an essential nutrient for healthy bones and teeth in both
RESULTS: The results of multivariate repeated measures analysis showed a change in serum 25-Hydroxycholecalciferol levels after vitamin D supplementation $(\mathrm{F}=296.132, p<0.05)$. There was also an indication of difference between the vitamin D group and placebo group. The only significant relationship was found between vitamin D and FBG $(p<0.05)$. Supplementation of vitamin D improved FBG but did not contribute to improving insulin level. There was no association between vitamin D supplementation and WHR.

CONCLUSION: This study concludes that supplementation of vitamin D improves FBG, but it has no contribution in improving insulin level. No association exists between vitamin D supplementation and WHR. The insignificant correlation of vitamin D intake with WHR and insulin can be attributed to the small sample size, therefore, further research is suggested with large sample size.

KEYWORDS: vitamin D, fasting blood glucose, body mass index, waist hip ratio, hypovitaminosis D

Indones Biomed J. 2019; 11(1): 42-7 adults and children.(1) As imperative vitamin D is for proper growth of human body, its deficiency can cause serious health concerns, out of which bone loss and bone fracture are prominent degrading consequences. Its deficiency may also lead to rickets, osteomalacia and osteoporosis in children, adults and women respectively.(2)

The principal source of the vitamin D in our body is cholecalciferol synthesis in the skin, which starts through direct sun exposure. The ultraviolet-B (UVB) light from the 
sun is absorbed by the skin that activates the synthesis of vitamin D. After its synthesis, it is regulated in the body by several factors which include intestinal absorption, renal function, serum calcium and parathyroid hormone (PTH) levels.(3) Any disturbance to aforementioned factors, from synthesis to regulation, can lead to the deficiency of vitamin D. In addition to these factors, other physical characteristics such as age, skinfold body thickness and weight also play significant role in vitamin D deficiency.(4) Since our skin is the main source of vitamin D synthesis, its pigmentation level also serves as an important factor in determining vitamin D concentration in the body.(5) For instance, darker skin (more pigmentation) hampers the absorption of UVB light from the sun into the skin (5-7), leading to reduced vitamin $\mathrm{D}$ synthesis and increasing the risk of its deficiency. (6) Due to an excessive skin pigmentation, Asian population is at more risk of developing vitamin D deficiency than white population.(8)

Another important factor that determines vitamin D status in the body is Serum 25-hydroxy vitamin D $(25(\mathrm{OH}) \mathrm{D})$ level. Its normal range is between 16 to $48 \mathrm{ng} /$ $\mathrm{mL}$ and its level under $8 \mathrm{ng} / \mathrm{mL}$ is considered to be the cause of rickets and osteomalacia.(7) Serum PTH levels are also important measures which determine vitamin D levels.(3) PTH is needed to stimulate bone mineralisation and it also increases the calcium concentration, needed for essential biological processes in the body such as muscle contraction and nerve conduction.(9)

Vitamin D deficiency is considered as one of the most common illnesses worldwide.(10) Vitamin D deficiency is involved in several medical conditions such as poor bone development, common cancers, cardiovascular disease and diabetes.(9-11) In Saudi Arabia, for example, a number of studies have reported that although there is plentiful sunlight and sufficient supplementary products are available in the market, vitamin D level is still very low among various sections of the population. $(3,7,12)$ An appreciable proportion $(23 \%)$ of women suffer from vitamin D deficiency at the age of 50 and above.(13)

Unfortunately, the incidence of vitamin D deficiency is also increasing amongst younger obese women. This alarming condition can be attributed to certain cultural reasons such as clothing which limits skin exposure to sunlight. Lifestyle also plays a role in causing vitamin D deficiency such as sedentary lifestyle and poor diet.(12) The association of vitamin D deficiency with obesity has already been established in the literature. For instance, in a recent study, there are factors leading to vitamin D deficiency in obesity include sedentary lifestyle, low sunlight exposure or less outdoor activity, and vitamin D metabolism derangement due to its deposition in adipose tissues, and volumetric dilution.(14)

In existing literature, most studies suggest that Vitamin D deficiency is more prevalent amongst women, teenagers and young children than men.(15) Among women, older age group is more likely to suffer from vitamin D deficiency manifested as osteoporosis and other systemic conditions such as diabetes.(16) Evidence also suggests that poor vitamin $\mathrm{D}$ level has been observed amongst younger women, which indicates that women in Saudi Arabia are developing serious conditions like osteoporosis before the age of menopause. $(3,7,12)$ The situation becomes more serious in obese patients who are already prone to or possess vitamin D deficiency. Interestingly, no clinical study has been reported in Saudi Arabia so far that provides further insights on the effect of vitamin D supplementation on obese young females.

Current study aims to examine the difference of vitamin D level between the participants in the experimental group who received vitamin $\mathrm{D}$ and the control group who received placebo (10 $\mathrm{mL}$ of normal saline drops). The study aims to examine the association between post supplementation of vitamin $\mathrm{D}$ and placebo on insulin, fasting blood glucose (FBG) and waist-hip ratio (WHR) in young and obese Saudi females with pre-existing hypovitaminosis D.

\section{Methods}

This study was conducted using prospective parallel randomised controlled clinical trial which was double blinded: patients and researchers. The pilot trial included 30 obese $\left(\right.$ BMI $\left.>30 \mathrm{~kg} / \mathrm{m}^{2}\right)$ medical students with age ranging from 18 to 23 years; they also had pre-exiting vitamin $\mathrm{D}$ deficiency (serum $25(\mathrm{OH}) \mathrm{D}<20 \mathrm{ng} / \mathrm{mL}$ ). Subjects who had a history of any endocrine disease (especially diabetes mellitus) or any systemic disease such as malabsorption, liver or renal disease were excluded from the study. Also excluded were the students who were pregnant and/ or lactating mothers, smokers, had a history of recent weight loss or who were already taking any medications or multivitamins. The study protocol was approved by the Research and Ethics Committee of King Fahd University Hospital (IRB No. KFHU/16-P012011).

Subjects were randomly assigned to either vitamin D group (receiving 50,000 IU/week of cholecalciferol drops) or Placebo group (receiving $10 \mathrm{~mL}$ of normal saline drops) for 12 weeks. A block randomization procedure with 
serial entry in blocks was used.(17) Four participants were included in each block, ensuring that two students within each block were allocated to vitamin D group and two were allocated to placebo group. Subjects were advised to maintain their usual diet and avoid taking any vitamin D supplementation on their own throughout the trial period. They were also asked to inform the researcher if any other medication would be prescribed to them for any health issue during the trail.

\section{Blood Sampling}

Venous blood samples were taken by a phlebotomist, using a sterile vacutainer. $10 \mathrm{~mL}$ of blood was collected from each participant $(9 \mathrm{~mL}$ was collected in plain vacutainers for the preparation of serum samples and $1 \mathrm{~mL}$ of blood was collected in vacutainers buffered with EDTA anticoagulant for all blood samples. All students were asked to fast overnight (12 $\mathrm{h}$ with no food or beverage except water).(18)

The blood was allowed to clot for $30 \mathrm{~min}$ after extraction and then centrifuged for $20 \mathrm{~min}$ at 3000 rpm within 2 h.(19) After centrifugation, the serum was separated, and transferred to an Eppendorf tube, marked with identification code on sticker and stored at $-80^{\circ} \mathrm{C}$ until the time of the analysis.

Fasting blood samples were obtained at the baseline (November 2016) and at the end of supplementation period of the study (February 2017). The FBG level was measured and recorded through Accu-Check Glucometer (Roche Diagnostics, Indianapolis, USA), vitamin D level was assessed by measuring serum 25(OH)D level through Enzyme-linked Immunosorbent Assay (ELISA), and Plasma insulin was measured through insulin ELISA kit. Insulin sensitivity was calculated by homeostasis model assessment of insulin resistance (HOMA-IR) by (fasting plasma glucose $x$ fasting insulin)/405.

\section{Statistical Analysis}

The data collected throughout the course of this study was analysed in order to draw a comparison between the vitamin $\mathrm{D}$ and placebo group. Mean and standard deviation of all variables was calculated. All analysis was performed using SPSS package 24 .

\section{Results}

\section{Descriptive Statistics of Vitamin and Placebo Groups}

This study took a sample of 30 individuals who are divided into two groups (15 vitamin D and 15 placebo). The baseline characteristics of all participants is shown in Table 1. Those individuals assigned to the vitamin $\mathrm{D}$ group received vitamin D supplement while the second group received placebo. Comparison of BMI and metabolic risk factors before and after treatment was Table 2, meanwhile the comparison of mean differences was shown in Table 3.

The results of the multiple regression model in Table 4 demonstrated no significant relationship between the supplementation of vitamin D (post) and the explanatory factors (WHR and insulin, $p>0.05$ ). The only significant relationship was found between vitamin $\mathrm{D}$ and FBG $(p<0.05)$.

Table 1. Baseline characteristics of all participants $(n=30)$.

\begin{tabular}{lccc}
\hline & \multicolumn{2}{c}{ Mean $( \pm$ SD $)$} & \\
\cline { 2 - 3 } & $\begin{array}{c}\text { Vitamin D } \\
(\mathrm{n}=15)\end{array}$ & $\begin{array}{c}\text { Placebo } \\
(\mathrm{n}=15)\end{array}$ & \\
\hline Age $($ years $)$ & $19.7( \pm 0.72)$ & $20.4( \pm 1.18)$ & 0.050 \\
BMI $\left(\mathrm{kg} / \mathrm{m}^{2}\right)$ & $35( \pm 3.85)$ & $32.2( \pm 2.99)$ & 0.032 \\
WHR & $0.8( \pm 0.04)$ & $0.8( \pm 0.06)$ & 0.355 \\
OHD $(\mathrm{ng} / \mathrm{mL})$ & $2.9( \pm 0.93)$ & $3( \pm 1.08)$ & 0.700 \\
FBG $(\mathrm{mmol} / \mathrm{L})$ & $88.2( \pm 7.42)$ & $85.4( \pm 8.81)$ & 0.354 \\
Insulin $(\mathrm{pmol} / \mathrm{L})$ & $5.1( \pm 12.47)$ & $6.3( \pm 12.75)$ & 0.811 \\
HOMA-IR & $1.1( \pm 2.84)$ & $1.2( \pm 2.26)$ & 0.967 \\
\hline
\end{tabular}

BMI: body mass index; WHR: waist to hip ratio; OHD: hydroxyl vitamin D; FBG: fasting blood glucose; HOMA-IR: homeostatic model assessment of insulin resistance. *Analyzed by independent sample T-test.

\section{Discussion}

This study aims to assess the impact of vitamin D supplementation on body parameters and metabolic factors such as WHR, insulin and FBG in obese young Saudi females.

The results demonstrated that the rise in FBG level in placebo group were more pronounced than incremental increase in the vitamin D group, as can be seen in Table 2. This significant finding suggests that vitamin $D$ has a potential role in controlling the level of plasma glucose. This is also in line with findings of Iyengar, et al., who reported clinical association between FBG and vitamin D levels. The authors stressed that vitamin D plays a critical role in regulating blood glucose level.(20) However, in another study, no relationship was found between vitamin D concentration and FBG, insulin or glucagon secretion.(21) According to the later, vitamin D levels and glucose control 
Table 2. Comparison of BMI and metabolic risk factors before and after treatment $(\mathrm{n}=30)$.

\begin{tabular}{|c|c|c|c|c|c|c|}
\hline & \multicolumn{3}{|c|}{ Vitamin D $(n=15)$} & \multicolumn{3}{|c|}{ Placebo $(n=15)$} \\
\hline & Baseline & 12 weeks & $p$-value* & Baseline & 12 weeks & $p$-value* \\
\hline WHR & $0.8( \pm 0.04)$ & $0.8( \pm 0.07)$ & 0.277 & $0.8( \pm 0.06)$ & $0.8( \pm 0.06)$ & 0.135 \\
\hline OHD (ng/mL) & $2.9( \pm 0.93)$ & $31.3( \pm 9.22)$ & 0.000 & $3( \pm 1.08)$ & $5.7( \pm 1.64)$ & 0.000 \\
\hline $\mathrm{FBG}(\mathrm{mmol} / \mathrm{L})$ & $88.2( \pm 7.42)$ & $91( \pm 5.84)$ & 0.208 & $85.4( \pm 8.81)$ & $96( \pm 5.21)$ & 0.001 \\
\hline Insulin (pmol/L) & $5.1( \pm 12.47)$ & $4.7( \pm 12.55)$ & 0.146 & $6.3( \pm 12.75)$ & $6.3( \pm 12.48)$ & 0.964 \\
\hline HOMA-IR & $1.1( \pm 2.84)$ & $1.1( \pm 3.04)$ & 0.629 & $1.2( \pm 2.26)$ & $1.6( \pm 3.15)$ & 0.218 \\
\hline
\end{tabular}

BMI: body mass index; WHR: waist to hip ratio; OHD: hydroxyl vitamin D; FBG: fasting blood glucose; HOMA-IR: homeostatic model assessment of insulin resistance. *Analyzed by paired T-test.

in healthy individuals are independent variables and they have no effect on each other.

The results of this study found no significant relationship between vitamin $\mathrm{D}$ and insulin resistance ( $p>0.05$ ). This finding is contrary to the findings of Nagpal and colleagues who reported inverse relationship between vitamin $\mathrm{D}$ concentration and insulin resistance.(22) The main reason for this finding is the obesity characteristic of our subjects as obesity is directly related to insulin resistance. $(23,24)$ Another reason for such finding could be the small sample size used in this trial. A larger population size could have produced different results.

Despite this, the association between the vitamin D supplementation and insulin resistance has remained controversial in the literature.(25-27) Most of these studies have used cross-sectional approach and recommended the randomised clinical trials design. This current study, through a randomised control clinical trial, showed no significant relationship between vitamin $\mathrm{D}$ levels and insulin resistance. On the contrary, several other studies

Table 3. Comparison of mean differences (post and pretreatment $)(n=30)$.

\begin{tabular}{|c|c|c|c|}
\hline & \multicolumn{2}{|c|}{$\begin{array}{c}\text { Mean Differences }( \pm \text { SD) } \\
\text { (Post }- \text { Pre) }\end{array}$} & \multirow{2}{*}{$p$-value } \\
\hline & Vitamin D & Placebo & \\
\hline WHR & $-0.02( \pm 0.1)$ & $-0.01( \pm 0.03)$ & 0.648 \\
\hline OHD (ng/mL) & $28.4( \pm 9)$ & $2.63( \pm 1.67)$ & 0.000 \\
\hline FBG (mmol/L) & $2.8( \pm 8.2)$ & $10.6( \pm 9.99)$ & 0.027 \\
\hline Insulin (pmol/L) & $-0.46( \pm 1.2)$ & $0.03( \pm 2.57)$ & 0.503 \\
\hline HOMA-IR & $-0.04( \pm 0.3)$ & $0.37( \pm 1.11)$ & 0.190 \\
\hline
\end{tabular}

BMI: body mass index; WHR: waist to hip ratio; OHD: hydroxyl vitamin D; FBG: fasting blood glucose; HOMA-IR: homeostatic model assessment of insulin resistance. *Analyzed by independent sample T-test. have found a clear association between vitamin $\mathrm{D}$ and insulin secretion. $(22,28)$

With regards to WHR variable, the multiple regression model indicated no relationship with vitamin $\mathrm{D}(p>0.05)$. The findings of current study are interesting because there are several studies which state otherwise. For instance, Wehr, et al., revealed negative correlation between the supplementation of vitamin D and WHR.(29) Similarly, another study reported that vitamin D supplementation in overweight and obese female adults was associated with lower WHR.(30) The present findings could be due to the small sample size used in the trial. Nonetheless, the insignificant relationship between vitamin D supplementation and WHR has also been reported previously by several other studies. $(24,31,32)$

Although, no significant relationship was found in the current study, Semba, et al., stressed that weight factors such as high BMI and high body skin thickness may be regarded as predictors of vitamin D levels among women.(33) Many studies who suggest a clear association between obesity and vitamin D status have reported that obesity causes alteration in vitamin $\mathrm{D}$ metabolism, leading to decreased vitamin $\mathrm{D}$ absorption in obese individuals. (34-36)

The positive correlation of vitamin $\mathrm{D}$ with $\mathrm{FBG}$ has huge clinical significance. This finding suggests that obese females who suffer from vitamin D deficiency are more prone to diabetes mellitus. Numerous studies have concluded similar findings by associating vitamin D deficiency with the higher occurrence rate of diabetes in non-obese individuals. $(37,38)$ Our study stresses on the issue that vitamin D deficiency may aggravate the existing condition or increase the chances of developing diabetes in young obese females. This conclusion has higher relevance to Saudi population as vitamin D deficiency is relatively common in them due to cultural norms. 
Table 4. Regression coefficients model.

\begin{tabular}{lccccc}
\hline & \multicolumn{2}{c}{$\begin{array}{c}\text { Unstandardized } \\
\text { Coefficients }\end{array}$} & $\begin{array}{c}\text { Standardized } \\
\text { Coefficients }\end{array}$ & T & Sig. \\
\cline { 2 - 4 } & Beta & Std. Error & Beta & & \\
\hline (Constant) & 107.195 & 62.081 & - & 1.727 & 0.097 \\
Post WHR & 5.264 & 45.702 & 0.023 & 0.115 & 0.909 \\
Post Insulin & 0.13 & 0.239 & 0.11 & 0.545 & 0.591 \\
Post FBG & 1.09 & 0.517 & 0.449 & 2.109 & 0.046 \\
\hline
\end{tabular}

WHR: waist to hip ratio; FBG: fasting blood glucose.

The limitations of the study include small sample size and short duration of the trial. The small sample size may be attributed to the high number of drop-outs from the study who were otherwise suitable for inclusion. It could be due to the lack of their perceived benefit in the trial, attendance issue, and/or fear of repeated blood sampling. Moreover, the recruited subjects had BMI $>30 \mathrm{~kg} / \mathrm{m}^{2}$, therefore, the results of this study may not be applicable to non-obese population. Lastly, in this trial, only Saudi females were primarily recruited which may reduce the generalizability of this trial to males or other ethnic groups.

\section{Conclusion}

It can be concluded from the results of this study that supplementation of vitamin D improves FBG but it has no contribution to improving insulin level. The study also concludes that no association exists between vitamin D supplementation and WHR.

\section{References}

1. Trivedi DP, Doll R, Khaw KT. Effect of four monthly oral vitamin D3 (cholecalciferol) supplementation on fractures and mortality in men and women living in the community: randomised double blind controlled trial. BMJ Br Med J. 2003; 326: 469-72

2. Lips $P$, van Ginkel FC, Jongen MJ, Rubertus F, van der Vijgh WJ, Netelenbos JC. Determinants of vitamin D status in patients with hip fracture and in elderly control subjects. Am J Clin Nutr. 1987; 46: $1005-10$

3. Eastell R, Barton I, Hannon RA, Chines A, Garnero P, Delmas PD. Relationship of early changes in bone resorption to the reduction in fracture risk with risedronate. J Bone Min Res. 2003; 18: 1051-6.

4. Korkor AB. Reduced binding of [3H]1,25-dihydroxyvitamin D3 in the parathyroid glands of patients with renal failure. N Engl J Med. 1987; 316: 1573-7.
5. Chan J, Jaceldo-Siegl K, Fraser GE. Serum 25-hydroxyvitamin D status of vegetarians, partial vegetarians, and nonvegetarians: The Adventist Health Study-2. Am J Clin Nutr. 2009; 89: 1686-92.

6. Aloia JF, Patel M, Dimaano R, Li-ng M, Talwar SA, Mikhail M, et al. Vitamin D intake to attain a desired serum 25-hydroxyvitamin D. Am J Clin Nutr. 2008; 87: 1952-8.

7. Heaney RP. Functional indices of vitamin D status and ramifications of vitamin D deficiency. Am J Clin Nutr. 2004; 25: 1706-9.

8. Ardawi M-SM, Sibiany AM, Bakhsh TM, Qari MH, Maimani AA High prevalence of vitamin D deficiency among healthy Saudi Arabian men: relationship to bone mineral density, parathyroid hormone, bone turnover markers, and lifestyle factors. Osteoporos Int. 2012; 23: 675-86.

9. Holick MF. High prevalence of vitamin D inadequacy and implications for health. Mayo Clin Proc. 2006; 81: 353-73.

10. Holick MF. Vitamin D deficiency. N Engl J Med. 2007; 357: 266-81.

11. Thomas MK, Lloyd-Jones DM, Thadhani RI, Shaw AC, Deraska DJ, Kitch BT, et al. Hypovitaminosis D in medical inpatients. N Engl J Med. 1998; 338: 777-83

12. Alsuwaida AO, Farag YMK, Sayyari AA Al, Mousa D, Alhejaili F, Al-Harbi A, et al. Epidemiology of chronic kidney disease in the kingdom of Saudi Arabia (SEEK-Saudi Investigators) - A Pilot Study. Saudi J Kidney Dis Transpl. 2010; 21: 1066-72.

13. Greer W, Ahmed M, Rifai A, Sandridge AL. Exploring the extent of postmenopausal osteoporosis among Saudi arabian women using dynamic simulation. J Clin Densitom. 2008; 11: 543-54.

14. Savastano S, Barrea L, Savanelli MC, Nappi F, Di Somma C, Orio F, et al. Low vitamin D status and obesity: Role of nutritionist. Rev Endocr Metab Disord. 2017; 18: 215-25.

15. Moussavi M, Heidarpour R, Aminorroaya A, Pournaghshband Z, Amini M. Prevalence of vitamin D deficiency in Isfahani high school students in 2004. Horm Res. 2005; 64: 144-8.

16. Hashemipour S, Larijani B, Adibi H, Javadi E, Sedaghat M, Pajouhi $\mathrm{M}$, et al. Vitamin D deficiency and causative factors in the population of Tehran. BMC Public Health. 2004; 4: 1-6.

17. Wamberg L, Cullberg KB, Rejnmark L, Richelsen B, Pedersen SB. Investigations of the anti-inflammatory effects of vitamin $\mathrm{D}$ in adipose tissue: results from an in vitro study and a randomized controlled trial. Horm Metab Res. 2013; 45: 456-62.

18. Tzotzas T, Vlahavas G, Papadopoulou SK, Kapantais E, Kaklamanou D, Hassapidou M. Marital status and educational level associated to obesity in greek adults: data from the national epidemiological survey. Bio Med Cent Public Heal. 2010; 10: 732. doi: 10.1186/14712458-10-732.

19. Salehpour A, Hosseinpanah F, Shidfar F, Vafa M, Razaghi M, Dehghani S, et al. A 12-week double-blind randomized clinical 
trial of vitamin $\mathrm{D}(3)$ supplementation on body fat mass in healthy overweight and obese women. Nutr J. 2012; 11: 78. doi: 10.1186/1475-2891-11-78.

20. Iyengar S, Hamman RF, Marshall JA, Majumder PP, Ferrell RE, Rao $\mathrm{DC}$, et al. On the role of vitamin D binding globulin in glucose homeostasis: results from the San Luis Valley Diabetes Study. Genet Epidemiol. 1989; 6: 691-8.

21. Kavaric S, Vuksanovic M, Bozovic D, Jovanovic M, Jeremic $\mathrm{V}$, Radojicic Z, et al. Body weight and waist circumference as predictors of vitamin $\mathrm{D}$ deficiency in patients with type 2 diabetes and cardiovascular disease. Vojnosanit Pregl. 2013; 70: 163-9.

22. Nagpal J, Pande JN, Bhartia A. A double-blind, randomized, placebo-controlled trial of the short-term effect of vitamin D3 supplementation on insulin sensitivity in apparently healthy, middle-aged, centrally obese men. Diabet Med. 2009; 26: 19-27.

23. Al-Sultan AI, Amin TT, Abou-Seif MA, Al Naboli MR. Vitamin D, parathyroid hormone levels and insulin sensitivity among obese young adult Saudis. Eur Rev Med Pharmacol Sci. 2011; 15: $135-47$.

24. Chiu KC, Chu A, Go VLW, Saad MF. Hypovitaminosis D is associated with insulin resistance and Beta cell dysfunction. Am J Clin Nutr. 2004; 25: 820-5.

25. Jackson RD, LaCroix AZ, Gass M, Wallace RB, Robbins J, Lewis $\mathrm{CE}$, et al. Calcium plus vitamin D supplementation and the risk of fractures. N Engl J Med. 2006; 354: 669-83.

26. Kayaniyil S, Gerstein HC, Veith R, Perkins BA, Retnakaran R, Harris $\mathrm{SB}$, et al. Association of vitamin $\mathrm{D}$ with insulin resistance and betacell dysfunction in subjects at risk for type 2 diabetes. Diabetes Care. 2010; 33: 1379-81.

27. Brenner DR, Arora P, Garcia-Bailo B. The relationship between metabolic syndrome and markers of cardiometabolic disease among Canadian adults. J Diabetes Metab. 2011; s2: 167-72.

28. Cigolini M. Serum 25-Hydroxyvitamin D3 concentrations and prevalence of cardiovascular disease among type 2 diabetic patients. Diabetes Care. 2006; 29: 722-4.
29. Wehr E, Pilz S, Schweighofer N, Giuliani A, Kopera D, Pieber TR, et al. Association of hypovitaminosis D with metabolic disturbances in polycystic ovary syndrome. Eur J Endocrinol. 2009; 161: 575-82.

30. Carrillo AE, Flynn MG, Pinkston C, Markofski MM, Jiang Y, Donkin $\mathrm{SS}$, et al. Impact of vitamin D supplementation during a resistance training intervention on body composition, muscle function, and glucose tolerance in overweight and obese adults. Clin Nutr. 2013; 32: $375-81$

31. Mahmoudi S, Brunet A. Aging and reprogramming: A two-way street. Curr Opin Cell Biol. 2012; 24: 744-56.

32. Faraji R, Sharami SH, Zahiri Z, Asgharni M, Kazemnejad E, Sadeghi S. Evaluation of relation between anthropometric indices and vitamin D concentrations in women with polycystic ovarian syndrome. J Fam Reprod Heal. 2014; 8: 123-9.

33. Semba RD, Garrett E, Johnson BA, Guralnik JM, Fried LP. Vitamin D deficiency among older women with and without disability. Am J Clin Nutr. 2000; 72: 1529-34.

34. Baradara A, Behradmanesh S, Nasri H. Association of body mass index and serum vitamin D level in healthy Iranian adolescents. Endokrynol Pol. 2012; 63: 29-33.

35. Need AG, Morris HA, Horowitz M, Nordin C. Effects of skin thickness, age, body fat, and sunlight on serum 25 -hydroxyvitamin D. Am J Clin Nutr. 1993; 58: 882-5.

36. Seiler C, Stoller M, Pitt B, Meier P. The human coronary collateral circulation: Development and clinical importance. Eur Heart J. 2013; 34: 2674-82.

37. Mouquet F, Cuilleret F, Susen S, Sautire K, Marboeuf P, Ennezat PV, et al. Metabolic syndrome and collateral vessel formation in patients with documented occluded coronary arteries: Association with hyperglycaemia, insulin-resistance, adiponectin and plasminogen activator inhibitor-1. Eur Heart J. 2009; 30: 840-9.

38. Yetkin E, Topal E, Erguzel N, Senen K, Heper G, Waltenberger J. Diabetes mellitus and female gender are the strongest predictors of poor collateral vessel development in patients with severe coronary artery stenosis. Angiogenesis. 2015; 18: 201-7. 